Article

\title{
Assessing the Impacts of Hierarchical Healthcare System on the Accessibility and Spatial Equality of Healthcare Services in Shenzhen, China
}

\author{
Zhuolin Tao ${ }^{1,2}$ and Wenchao Han ${ }^{3, *}$ \\ 1 Faculty of Geographical Science, Beijing Normal University, No.19, Xinjiekouwai Ave., Haidian, \\ Beijing 100875, China; taozhuolin@bnu.edu.cn \\ 2 Key Laboratory of Regional Sustainable Development Modelling, Institute of Geographic Sciences and \\ Natural Resources Research, Chinese Academy of Sciences, Chaoyang District, Beijing 100101, China \\ 3 Chinese Research Academy of Environmental Sciences, Beijing 100012, China \\ * Correspondence: 201631490013@mail.bnu.edu.cn
}

check for updates

Citation: Tao, Z.; Han, W. Assessing the Impacts of Hierarchical Healthcare System on the Accessibility and Spatial Equality of Healthcare Services in Shenzhen, China. ISPRS Int. J. Geo-Inf. 2021, 10, 615. https://doi.org/ 10.3390/ijgi10090615

Academic Editor: Wolfgang Kainz

Received: 23 June 2021

Accepted: 13 September 2021

Published: 16 September 2021

Publisher's Note: MDPI stays neutral with regard to jurisdictional claims in published maps and institutional affiliations.

Copyright: (c) 2021 by the authors. Licensee MDPI, Basel, Switzerland. This article is an open access article distributed under the terms and conditions of the Creative Commons Attribution (CC BY) license (https:// creativecommons.org/licenses/by/ $4.0 /)$.

\begin{abstract}
The hierarchical healthcare system is widely considered to be a desirable mode of the delivery of healthcare services. It is expected that the establishment of a hierarchical healthcare system can help provide better and more equal healthcare accessibility. However, limited evidence has been provided on the impacts of a hierarchical healthcare system on healthcare accessibility. This study develops an improved Hierarchical two-step floating catchment area (2SFCA) method, which incorporates variable catchment area sizes, distance friction effects and utilization efficiency for facilities at different levels. Leveraging the Hierarchical 2SFCA method, various scenarios are set up to assess the accessibility impacts of a hierarchical healthcare system. The methods are applied in a case study of Shenzhen. The results reveal significant disparity and inequality in healthcare accessibility and also differences between various facility levels in Shenzhen. The overall healthcare accessibility and its equality can be significantly improved by fully utilizing existing facilities. It is also demonstrated that allocating additional supply to lower-level facilities can generate larger accessibility gains. Furthermore, allocating new supply to primary facilities would mitigate the inequality in healthcare accessibility, whereas inequality tends to be aggravated with new supply allocated to tertiary facilities. These impacts cannot be captured by traditional accessibility measures. This study demonstrates the pivotal role of primary facilities in the hierarchical healthcare system. It can contribute to the literature by providing transferable methods and procedures for measuring hierarchical healthcare accessibility and assessing accessibility impacts of a hierarchical healthcare system in developing countries.
\end{abstract}

Keywords: healthcare service; hierarchical system; accessibility; equality; 2SFCA; China

\section{Introduction}

Human health is a key topic drawing great attention from global researchers from domains such as medicine, public health, urban planning and geography. With the increasing improvement in wealth and well-being, increasing attention is being paid to health. The United Nations highlights good health and well-being as one of the 17 Sustainable Development Goals [1]. In China, the "Healthy China 2030" strategy emphasizes the promotion of the equalization of health services and thus the health status of residents [2].

The rational distribution of healthcare facilities is a critical influencing factor on the utilization and outcomes of healthcare services [3,4]. Inequality in accessing healthcare services is considered to be a major threat to the overall level of health $[3,5]$. Even in developed countries such as the U.S. where the total supply of healthcare services is relatively sufficient, there is still a considerable proportion of the population lacking access to healthcare services due to the unequal distribution of resources [6]. In developing 
countries, the provision of healthcare services is facing great challenges in terms of both insufficient supply and an unbalanced distribution of healthcare services [7-10]. In China, the difficulty in seeking a doctor ("kanbingnan" in Chinese) has been a recurrent topic drawing attention from both policymakers and researchers. The unequal distribution of healthcare resources is considered to be a key cause of this problem [11].

The delivery of healthcare services is generally organized in a hierarchical manner [12-14]. In other words, there are multi-level facilities that provide differentiated healthcare services to patients. Generally, higher-level facilities provide more complicated diagnoses and treatments for difficult miscellaneous diseases, while lower-level facilities are responsible for common diseases and rehabilitation treatments $[15,16]$. A well-established hierarchical healthcare system is crucial for the improvement of the efficiency and equality of healthcare services. The "kanbingnan" problem in China has often been attributed to the disordered and underdeveloped hierarchical healthcare system [11,13]. A major feature of this system is that high-level facilities are heavily overloaded, whereas low-level facilities remain underutilized. Therefore, the establishment of a hierarchical healthcare system is highlighted in the "Healthy China 2030" strategy [2]. The outbreak of the COVID-2019 pandemic has further uncovered the weakness of primary healthcare services in China [17], which makes an urgent appeal for a better hierarchical healthcare system.

Spatial accessibility is a useful tool that has been widely used to evaluate the configuration of public facilities, including healthcare facilities. It measures how many opportunities people can access and how easy it is to access these opportunities [18]. Spatial accessibility is mainly determined by the amount of available resources and the spatial barriers that patients have to overcome to seek healthcare services $[3,19,20]$. These factors are closely related to population distribution, facility distribution and the transport network, which are common components dealt with in urban planning. Therefore, spatial accessibility is an appropriate planning and policy countermeasure to promote the equality of healthcare resources and improve health outcomes.

Numerous methods have been developed for measuring spatial accessibility. Basic measures include the proximity-based method, supply-demand ratio method and the cumulative opportunities method. The proximity-based method focuses on the travel cost from each location to the nearest facility [4]. The supply-demand ratio method calculates the ratio of total supply to total demand within each geographic unit (usually represented by administrative or census units), which reflects the availability of services [20]. The cumulative opportunities method measures the amount of opportunities that can be reached within a certain distance or travel time [21]. However, some essential factors are overlooked in these basic accessibility measures, e.g., the complex interaction between demand and supply sides and the competition effects among demanders. More comprehensive measures have been proposed to better capture various factors, among which the two-step floating catchment area (2SFCA) method and its numerous variants are the most widely used [3,22,23]. Essentially, 2SFCA is a special form of gravity-based measures [24]. A series of improved forms of 2SFCA have been developed, including the Enhanced 2SFCA [25], Gaussian 2SFCA [26], Nearest-Neighbor 2SFCA [27], Modified 2SFCA [28], Multi-modal 2SFCA [29] and iFCA [22], which further strengthen the prevalence of the 2SFCA method family.

Until recently, however, few studies have strived to measure healthcare accessibility in a hierarchical system. Some consensuses have been reached among these exploratory studies on the heterogeneity in catchment area sizes, distance friction effects and transport modes among different levels [12,15,30,31]. Meanwhile, some studies are focused on the impacts of the referrals between facilities at different levels on healthcare accessibility $[13,32,33]$. Furthermore, the establishment of a hierarchical medical system would also influence the health seeking behaviors of patients [16]. Notably, most existing studies on the impacts of a hierarchical healthcare system on healthcare accessibility rely on the 2SFCA method. As pointed out by Tao et al. [15], however, 2SFCA and its variants are defective in measuring accessibility to hierarchical facilities. Traditional methods treat distance decay effects in a relative form but overlook the absolute form of distances [28]. As 
a result, the differences in the travel distance to facilities at various levels are excluded in the estimated accessibility. Consider two scenarios where the same amounts of healthcare resources are configured at different levels. The resulting accessibility in the scenario where the resources are assigned to lower-level facilities should be higher because there are generally more lower-level facilities and they can be accessed by patients at a shorter distance. However, the traditional 2SFCA methods would generate the same accessibility in these two scenarios [15]. Furthermore, it remains difficult to differentiate the heterogenous accessibility impacts of allocating healthcare resources at different levels. This drawback would undoubtedly lead to biases in the understanding of the accessibility impacts of a hierarchical healthcare system.

Given the above, this study intends to evaluate the impacts of the establishment of a hierarchical healthcare system on healthcare accessibility and its equality in China. Our focus is on the differences in resource utilization rates and accessibility improvement among healthcare facilities at different levels. The evaluation is conducted by applying and adopting the Hierarchical 2SFCA (H2SFCA) method. H2SFCA was developed by Tao et al. [15], and it incorporates multi-level catchment areas and distance decay functions and both relative and absolute distance decay effects into the traditional 2SFCA framework. This study aims to further improve H2SFCA by adjusting the absolute distance decay function to more accurately reflect the hierarchical nature of healthcare accessibility. Leveraging this improved accessibility measure, this study will evaluate the accessibility impacts of a hierarchical healthcare system in two different scenarios. In the first scenario, it is assumed that the establishment of a hierarchical healthcare system would improve the utilization rates of existing resources provided by low-level facilities, based on which the accessibility impacts are assessed. In the second scenario, we further investigate the differences in accessibility improvement by allocating resources to facilities at different levels. The methods and procedures will be applied and validated in a case study of Shenzhen. This study can strengthen our understanding of the accessibility impacts of a hierarchical healthcare system and provide evidence for relevant policymaking in China. In addition, it can also provide transferable methods and procedures for measuring accessibility to hierarchical facilities and evaluating accessibility impacts of hierarchical healthcare systems in other developing countries.

\section{Materials and Methods}

Shenzhen, one of the mega-cities in China, is selected as the study area. Shenzhen has a typical Chinese hierarchical healthcare system [15,30]. As one of the earliest economic special zones in China, Shenzhen has experienced rapid economic growth and urbanization since the opening-up. The healthcare service system in Shenzhen, however, lags behind its economic success. By 2019, the total population of Shenzhen was 13.44 million, residing on a land area of 1997 square kilometers.

\subsection{Hierarchical Healthcare System in China}

Public healthcare facilities are organized as a three-level hierarchical system in China, including tertiary hospitals, secondary hospitals and primary facilities from the highest to the lowest level [34]. Primary healthcare facilities mainly refer to community healthcare service centers (CHSCs) in Chinese cities, which are responsible for the basic treatments and rehabilitation of common diseases, frequently occurring diseases and chronic diseases. Tertiary and secondary hospitals provide emergency treatments, hospitalization, and diagnoses and treatments of difficult miscellaneous diseases. Medical scientific research and teaching and the development of key medical disciplines are mainly organized in tertiary hospitals. Referrals between different levels are essential for the operation of the hierarchical system. Theoretically, most treatments should be performed at primary facilities.

Generally, hierarchical facilities can be classified into single-flow versus multi-flow, nested versus non-nested or spatially coherent systems versus non-coherent systems $[15,35,36]$. Taking the three-level healthcare facilities as an example, in a single-flow system, patients 
must be assigned to primary facilities to receive primary treatments, and then transferred to higher-level facilities if needed. By contrast, patients can choose facilities at any level at the first step in a multi-flow system. In a nested system, higher-level hospitals can also provide primary healthcare services, while in a non-nested system facilities at different levels provide mutually exclusive services and primary treatments can only be provided at primary facilities. A spatially coherent system refers to the system where the service scopes of facilities at different levels are consistent. In other words, patients assigned to a lower-level facility can only be transferred to the higher-level facility that this facility is affiliated with. In a spatially non-coherent system, however, there are no such constraints on health seeking behaviors.

The Chinese hierarchical healthcare system is currently not well established, and it can be classified as a multi-flow, nested and spatially non-coherent system [15]. A large proportion of patients seek healthcare at higher-level facilities, especially at tertiary hospitals, partially due to the unbalanced service capacities and medical levels among different levels. As a result, higher-level facilities, especially tertiary hospitals, are highly busy and even overloaded, whereas lower-level facilities are unoccupied to a certain extent [13].

It is natural to expect that the establishment of a hierarchical healthcare system would improve the utilization efficiency of lower-level healthcare resources by attracting more patients to choose lower-level facilities. It has been confirmed that the introduction of the hierarchical healthcare system has increased the utilization of primary facilities in some Chinese cities [16]. However, it remains unknown how the establishment of a hierarchical healthcare system and increases in utilization efficiency of lower-level facilities would influence healthcare accessibility.

\subsection{Data Sources}

The analysis units in this study are set as the communities, which are the most basic administrative and census units in Chinese cities. Based on the 6th national population census of China, there were 771 communities in Shenzhen, each of which had an average population of 13.4 thousand persons and an average area of 2.59 square kilometers. Most healthcare accessibility studies in China used town-level units in their analyses. The communities used in this study are much finer than town-level units and can provide more detailed and precise results. The town-level population density and communities are shown in Figure 1.

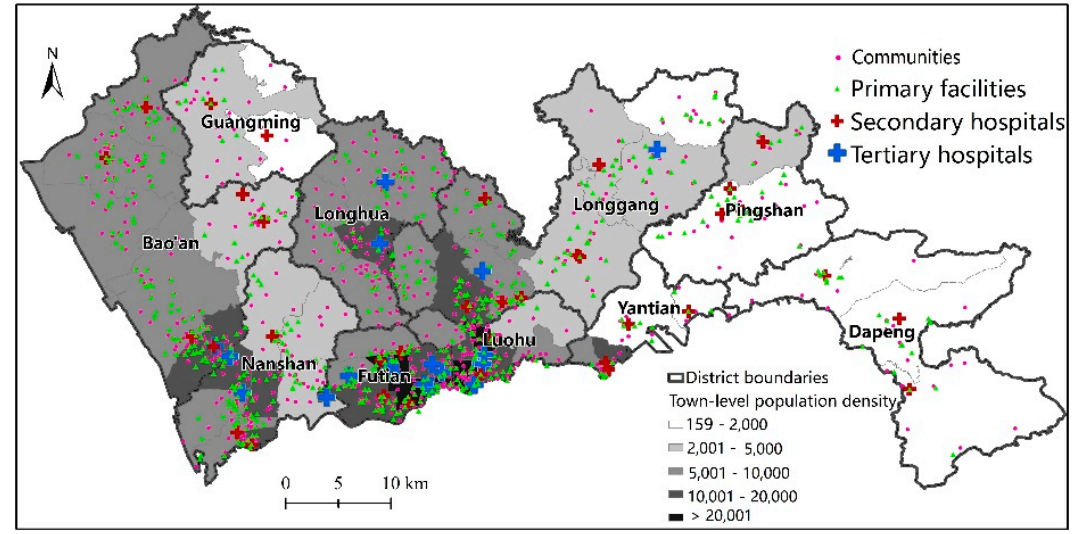

Figure 1. The distribution of communities, town-level population density and hierarchical healthcare facilities in Shenzhen.

The public healthcare facilities data were obtained from the official website of the Shenzhen Municipal Health Commission [37], including the name, grade, number of physicians and beds and address of each public healthcare facility. The addresses were then transferred to geographic coordinates by using Baidu Map Geocoding API (Application 
Programming Interface). The numbers of primary facilities, secondary hospitals and tertiary hospitals are 612, 35 and 19, respectively. The supply sizes of healthcare facilities are represented by their physicians. The average sizes of the three-level facilities are 6,182 and 422 physicians, respectively. As shown in Figure 1, primary and secondary facilities are relatively dispersed across the districts, whereas tertiary hospitals are mainly concentrated in the central districts, e.g., Luohu, Futian and Nanshan.

The numbers of treatments at multi-level facilities were obtained from the "Summary of Health Statistics in 2019 of Shenzhen" [38]. This data source only includes CHSCs and general hospitals, without differentiation between tertiary and secondary hospitals. Therefore, the differences in utilization efficiency between tertiary and secondary hospitals were further estimated based on the average utilization rates of hospitalization beds. These data were obtained from the "2013 Yearbook of Health Statistics of Shenzhen" [39], which was the latest version of this yearbook that reports the bed utilization rates.

\subsection{Methods}

\subsubsection{The Gaussian 2SFCA}

The traditional 2SFCA has been questioned due to its treatment of identical accessibility within the catchment area of a facility $[3,25]$. Therefore, a series of improvements have been developed by existing studies by incorporating additional distance friction functions into the 2SFCA framework [3,22]. Among these, the Gaussian 2SFCA equipped with a Gaussian-form distance friction function has been widely applied $[5,26]$. The Gaussian 2SFCA can be expressed by Equations (1) and (2).

$$
A_{i}=\sum_{j} \frac{S_{j} f\left(d_{i j}\right)}{\sum_{k} P_{k} f\left(d_{k j}\right)}
$$

where $A_{i}$ is the accessibility at $i$-th location, $S_{j}$ is the supply size of $j$-th facility, $P_{k}$ is the population at $k$-th location and $d_{i j}$ and $d_{k j}$ are travel times from $i$-th and $k$-th demand location to $j$-th facility, respectively. $f$ is the Gaussian-form distance friction function, which can be written as:

$$
f\left(d_{i j}\right)=\left\{\begin{array}{c}
\frac{e^{-1 / 2 \times\left(d_{i j} / d_{0}\right)^{2}}-e^{-1 / 2}}{1-e^{-1 / 2}}, \quad d_{i j} \leq D_{0} \\
0, \quad d_{i j}>D_{0}
\end{array}\right.
$$

where $D_{0}$ is the catchment area size of the facilities.

\subsubsection{The Hierarchical 2SFCA}

The traditional 2SFCA and gravity-based methods have a so-called weighted-average property, i.e., the population-weighted average accessibility of all units always equals the ratio of total supply to total population $[24,40]$. It means that the calculation of accessibility is to allocate the resources of each facility to the demanders within its catchment area according to numbers of demanders and the travel costs to the facility in a relative sense. This property makes the accessibility results more easy to interpret and implement. However, as pointed out by existing studies $[15,28]$, this property would lead to the relative distance bias problem because only the relative distances rather than absolute distances work in the calculation of accessibility. Briefly speaking, the absolute distance means the absolute value of distance between communities and facilities, which is expressed in the form of travel time in this study. By contrast, the relative distance means the value or weight relative to the threshold distance (i.e., catchment area size). Therefore, when applied to the case of hierarchical facilities, traditional methods would generate biased accessibility results, especially in the comparison between accessibility at different levels [15]. It is urgently suggested that both relative and absolute distance friction effects should be considered in the measurement of hierarchical healthcare accessibility [15].

A Hierarchical 2SFCA (H2SFCA) has been proposed by Tao et al. [15], which is based on Gaussian 2SFCA and further incorporates multiple catchment area sizes and different 
distance friction effects for facilities at different levels. Enlightened by the Modified 2SFCA [28], an additional distance friction function is added into H2SFCA to account for both relative and absolute distance friction effects. However, Tao et al. set different catchment area sizes for facilities at different levels [15], just like the relative distance friction function. This study will improve H2SFCA by using a unified catchment area size for all facilities in the absolute distance friction function. By doing so, the differences in absolute distance friction effects between different facility levels can be accurately captured.

Furthermore, to reflect the increases in the utilization efficiency of low-level facilities induced by the hierarchical healthcare system, a variable representing the utilization efficiency of facilities is incorporated into the model. The combination of $S_{j}$ and $U_{l}$ represents the actual available supply of facility $j$ at level $l$. The improved H2SFCA can be written as:

$$
A_{i}=\sum_{l} \sum_{j \in\left\{d_{i j} \leq D_{l}\right\}} \frac{s_{j} U_{l} f r\left(d_{i j}\right) f a\left(d_{i j}\right)}{\sum_{k \in\left\{d_{k j} \leq D_{l}\right\}} P_{k} f r\left(d_{i j}\right)}
$$

where $A_{i}$ is the overall accessibility of unit $i$ in a hierarchical system, $l$ represents the level that a facility belongs to, $U_{l}$ is the utilization efficiency of facilities at level $l, D_{l}$ is the catchment area size at level $l$ and $f r$ and $f a$ are Gaussian-form functions reflecting relative and absolute distance friction effects, respectively. Other variables are the same as Equation (1). fr and $f a$ can be expressed as:

$$
\begin{gathered}
f r\left(d_{i j}\right)=\left\{\begin{array}{c}
\frac{e^{-1 / 2 \times\left(d_{i j} / D_{l}\right)^{2}}-e^{-1 / 2}}{1-e^{-1 / 2}}, \quad d_{i j} \leq D_{l} \\
0, d_{i j}>D_{l}
\end{array}\right. \\
f a\left(d_{i j}\right)=\left\{\begin{array}{cc}
\frac{e^{-1 / 2 \times\left(d_{i j} / D_{\max }\right)^{2}}-e^{-1 / 2}}{1-e^{-1 / 2}}, d_{i j} \leq D_{\max } \\
0, d_{i j}>D_{\text {max }}
\end{array}\right.
\end{gathered}
$$

where $f r$ specifies a variable catchment area size according to facility levels, while fa uses a unified catchment area size (i.e., the maximum value of $D_{l}$ ) for different facility levels. Specifically, $D_{\max }$ is set as the catchment area size of tertiary hospitals in this study.

According a survey conducted by Cheng et al. [41], travels to general hospitals in Chinese cities mainly rely on driving, while travels to primary healthcare facilities mainly rely on active modes including cycling or walking. The travel times between communities and general hospitals (tertiary and secondary) were estimated by using Baidu Map Navigation Application Programming Interface (API). This approach has superiority in terms of real-time traffic status, actual driving rules and updated road networks, and therefore can generate a reliable and accurate estimation of travel time [42,43]. The cycling mode travel times from communities to primary facilities were estimated based on the Euclidean distances and typical cycling speed. The latter was set as $9 \mathrm{~km} / \mathrm{h}$ according the " 2017 White Book of Shared Bikes and Urban Development in China" [44]. The geometric centroids of communities and healthcare facilities were set as origins and destinations, respectively.

As suggested by existing studies [5,45], the catchment area sizes were set based on the natural break points in the distribution of the travel time from each community to its nearest facility. By doing so, the setting of catchment areas ensures that most communities are included in the catchment areas, while the rest of the communities that are very distant from facilities are excluded from catchment areas and assigned with zero accessibility. The procedures were separately conducted for each facility level. After applying the procedures, the catchment area sizes for primary facilities, secondary hospitals and tertiary hospitals, i.e., $D_{1}, D_{2}$ and $D_{3}\left(D_{\max }\right)$, were set as 25,40 and $70 \mathrm{~min}$, respectively. 
2.3.3. Framework for Evaluating the Accessibility Impacts of Hierarchical Healthcare System

Based on scenario analyses of accessibility measured by H2SFCA, the impacts of a hierarchical healthcare system on healthcare accessibility and its equality can be quantified. In this study, two types of accessibility impacts will be examined.

The first type of accessibility impact (improving-efficiency accessibility impact) is induced by the improvement of low-level facilities' utilization efficiency. As demonstrated above, the resources at lower-level healthcare facilities are not fully utilized in the status quo healthcare system in China. The establishment of a hierarchical healthcare system would improve the hierarchy structure of the healthcare system and increase the utilization efficiency of low-level facilities. The first objective of this study is to evaluate this type of accessibility impact of the hierarchical healthcare system. Analyses will also be conducted to explore how such an accessibility impact would be distributed across the city.

The utilization efficiency is estimated based on the total treatments and physicians data. The average number of treatments per physician for CHSCs (i.e., primary facilities) and general hospitals (including both tertiary and secondary hospitals) is 1333 and 3595, respectively. To further differentiate the difference in utilization efficiency between tertiary and secondary hospitals, the average bed utilization rates were collected, which are $87 \%$ and $95 \%$, respectively. Considering that our main purpose is to examine the differences in utilization efficiency and accessibility gains between different levels, the utilization efficiency of tertiary hospitals is set as the benchmark. In other words, the actual utilization efficiency of tertiary hospitals is set as $100 \%$. The actual utilization efficiency of secondary hospitals and primary facilities is set as $90 \%$ and $40 \%$. By contrast, in the improved scenario, the utilization efficiency of facilities at all levels is supposed to increase to $100 \%$ due to the establishment of a hierarchical healthcare system. It can be observed from Table 1 that there is significant disparity in the utilization efficiency of facilities at different levels. Notably, the utilization efficiency of primary facilities is much lower than higher-level facilities. In the improved scenario where hierarchical healthcare was established, the service capacities of primary facilities and secondary hospitals can be increased by $150 \%$ and $11 \%$ compared to the status quo, which are equivalent to 1469 and 637 more physicians, respectively.

Table 1. Utilization efficiency of three-level healthcare facilities in actual and improved scenarios.

\begin{tabular}{ccc}
\hline \multirow{2}{*}{ Levels } & \multicolumn{2}{c}{ Utilization Efficiency } \\
\cline { 2 - 3 } & Actual & Improved \\
\hline Primary & $40 \%$ & $100 \%$ \\
Secondary & $90 \%$ & $100 \%$ \\
Tertiary & $100 \%$ & $100 \%$ \\
\hline
\end{tabular}

The second type of accessibility impact (adding-supply accessibility impact) is engendered in hypothetical scenarios where a certain newly added supply (physicians) was configured at different levels. The purpose is to examine the difference in accessibility gains of allocating the same amount of supply to facilities at different levels. Our expectation is that allocating supply to lower-level facilities would generate larger accessibility gains because lower-level facilities are closer to patients on average and thus can provide better accessibility. As for each level, it is assumed that the newly added supplies were equally allocated to each facility at this level. As a preliminary attempt, we assume that 2000 additional physicians in total were added in each scenario.

To measure and compare the inequality of healthcare accessibility in various scenarios, two popular metrics, namely the Gini coefficient and the coefficient of variation (CV), are applied. The Gini coefficient measures the extent to which the distribution of an indicator deviates from the equal distribution. $\mathrm{CV}$ is calculated by dividing the standard deviation by the mean value. Larger values of the Gini coefficient and CV both indicate a higher inequality. Moreover, both metrics can be applied for the comparison between different indicators. 
Figure 2 summarizes the procedures to conduct all the analyses in this study.

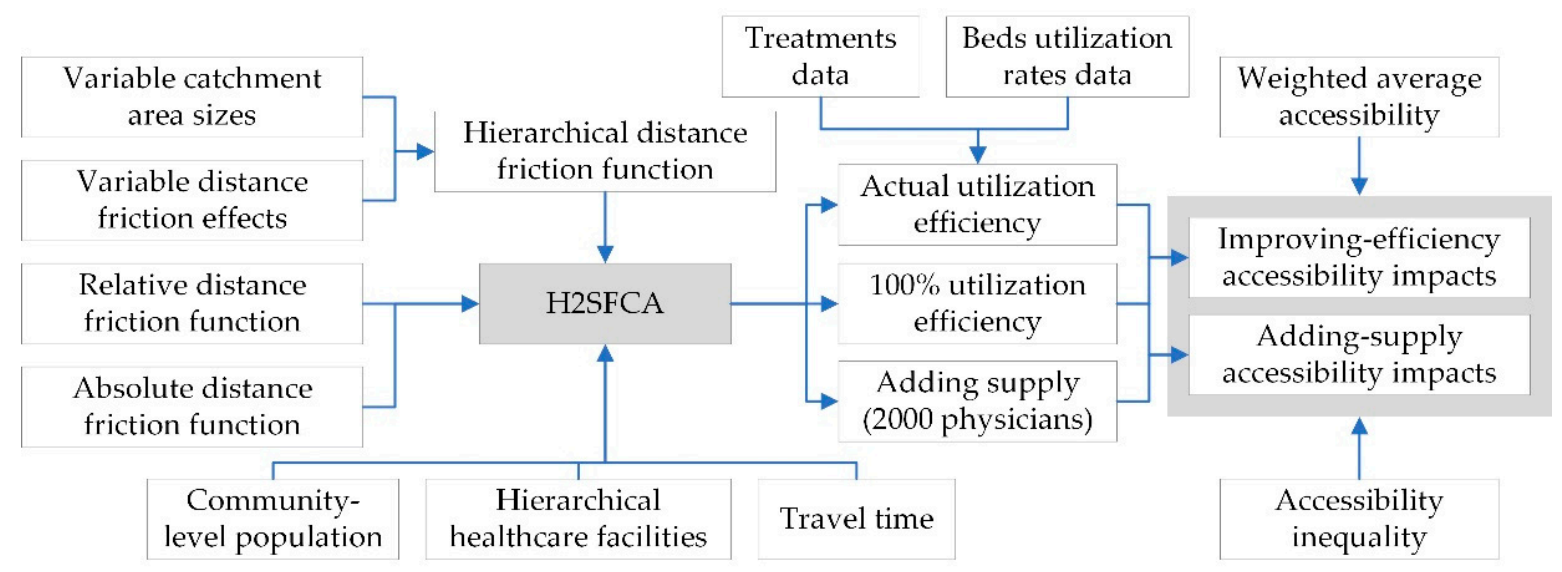

Figure 2. The framework of this study.

\section{Results}

\subsection{Actual Accessibility to Hierarchical Healthcare Facilities in Shenzhen}

We first measured the actual accessibility to hierarchical healthcare facilities under the actual utilization efficiency scenario, which reflects the actual available healthcare resources in the current healthcare system in Shenzhen. The values of accessibility indicate the average number of physicians available to each person. Figure 3 shows the overall actual healthcare accessibility that sums up the accessibility to three-level facilities. The intervals used for mapping accessibility were determined by the natural breaks approach. It can be first observed that there is a significant spatial inequality in the actual healthcare accessibility in Shenzhen. The overall accessibility is highest in Futian, one of the central districts in Shenzhen, and gradually decreases outwards from Futian. The second feature is that the accessibility in the eastern part is relatively higher than that in the western part of Shenzhen. It is noteworthy that the density of healthcare facilities is similar in eastern and western Shenzhen. The higher accessibility in eastern Shenzhen may be caused by its lower population density. This reveals considerable spatial mismatch between healthcare resource supply and demand in Shenzhen.

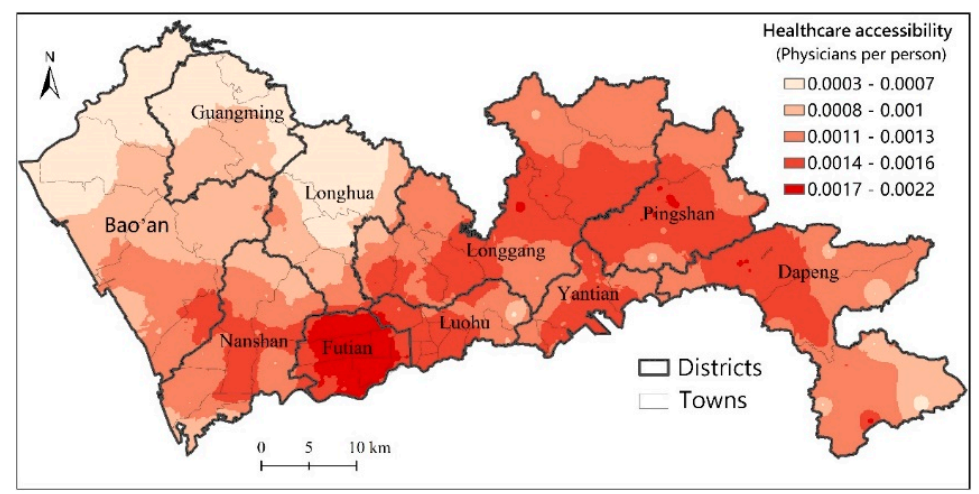

Figure 3. Actual overall healthcare accessibility in Shenzhen.

The improved H2SFCA method can also generate healthcare accessibility at each level. As shown in Figure 4, the magnitudes and distributions of actual healthcare accessibility at three levels are quite different. First, the magnitude of healthcare accessibility to primary facilities is much lower than accessibility to higher-level facilities. This demonstrates that though there is a large number of primary facilities, their service capacity and resources are still highly insufficient. 


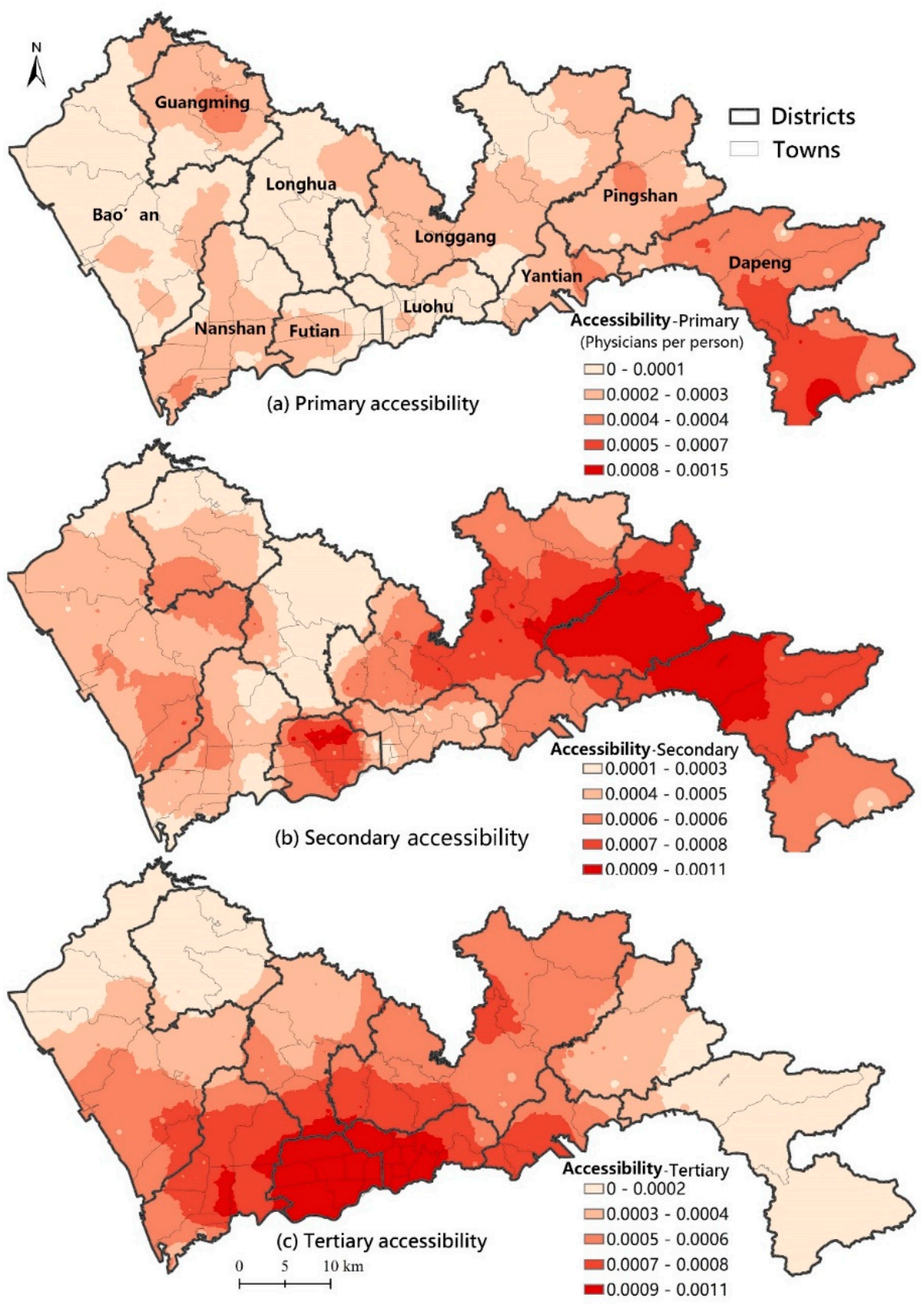

Figure 4. Actual accessibility to hospitals at various levels in Shenzhen.

Second, the distributions of healthcare accessibility at different levels are quite different. The accessibility to primary facilities is relatively equal across the city, which is also confirmed by the low CV value of 0.228 (Table 2). By contrast, the distribution of accessibility to secondary hospitals is more unbalanced, with a CV of 0.439 . It is higher in Futian, Longgang, Pingshan and Dapeng, but much lower in Longhua. The CV of accessibility to tertiary hospitals is 0.542 , indicating that the inequality in accessibility to tertiary hospitals is the largest. The accessibility to tertiary hospitals is highest in Futian and Luohu and rapidly decreases outwards. Briefly speaking, the distribution of accessibility to higher-level facilities is unequal, whereas the accessibility to primary facilities is equally distributed but is overall insufficient. 
Table 2. Improving-efficiency accessibility impacts of hierarchical healthcare system in Shenzhen.

\begin{tabular}{|c|c|c|c|c|c|c|c|c|c|}
\hline \multirow{2}{*}{ Levels } & \multicolumn{3}{|c|}{ Weighted Average Accessibility } & \multicolumn{3}{|c|}{$\begin{array}{l}\text { Accessibility Inequality } \\
\text { (CV) }\end{array}$} & \multicolumn{3}{|c|}{$\begin{array}{l}\text { Accessibility Inequality } \\
\text { (Gini) }\end{array}$} \\
\hline & Actual & $\begin{array}{l}\text { Improving- } \\
\text { Efficiency }\end{array}$ & Changes & Actual & $\begin{array}{l}\text { Improving- } \\
\text { Efficiency }\end{array}$ & Changes & Actual & $\begin{array}{l}\text { Improving- } \\
\text { Efficiency }\end{array}$ & Changes \\
\hline Overall & 0.00115 & 0.00140 & $21.7 \%$ & 0.338 & 0.320 & $-5.3 \%$ & 0.190 & 0.178 & $-6.3 \%$ \\
\hline Primary & 0.00014 & 0.00034 & $142.9 \%$ & 0.228 & 0.219 & $-3.9 \%$ & 0.248 & 0.236 & $-4.8 \%$ \\
\hline Secondary & 0.00048 & 0.00053 & $10.4 \%$ & 0.439 & 0.435 & $-0.9 \%$ & 0.240 & 0.236 & $-1.7 \%$ \\
\hline Tertiary & 0.00054 & 0.00054 & 0 & 0.542 & 0.542 & 0 & 0.311 & 0.311 & 0 \\
\hline
\end{tabular}

\subsection{Improving-Efficiency Accessibility Impacts of Hierarchical Healthcare System in Shenzhen}

The establishment of a hierarchical healthcare system would improve the utilization efficiency of existing resources of healthcare facilities, especially low-level facilities. This is expected to generate accessibility gains and also change the equality of accessibility. By setting the utilization efficiency of all facilities as $100 \%$ and comparing the resulting accessibility with the actual accessibility, the improving-efficiency accessibility impacts of a hierarchical healthcare system can be quantified. As shown in Table 2, the improvement in utilization efficiency can cause a $21.7 \%$ increase in the weighted average overall accessibility. Notably, the accessibility to primary facilities is greatly increased by $142.9 \%$. As for the impacts on accessibility equality, the CVs and Gini coefficients of accessibility were calculated.

Figure 5 shows the distribution of the changes in overall healthcare accessibility caused by the improvement in utilization efficiency, which is represented by the ratio of the after-improvement accessibility to the actual accessibility without the improvement. The accessibility ratio is larger than 1 across all units in Shenzhen, indicating that all communities can benefit from the improvement in utilization efficiency of existing facilities and the establishment of a hierarchical healthcare system. However, such accessibility gains are not evenly distributed as they are larger in peripheral areas, especially in the northwestern and southeastern parts of Shenzhen (i.e., Guangming and Dapeng districts). The accessibility gains are negatively correlated with the actual healthcare accessibility. Therefore, it is possible that an improvement in utilization efficiency can mitigate the inequality in healthcare accessibility. This can be clearly proved by the CVs presented in Table 2. The CVs of the overall, primary and secondary healthcare accessibility decreased by $5.3 \%, 3.9 \%$ and $0.9 \%$, respectively. The comparisons of Gini coefficients yield similar conclusions.

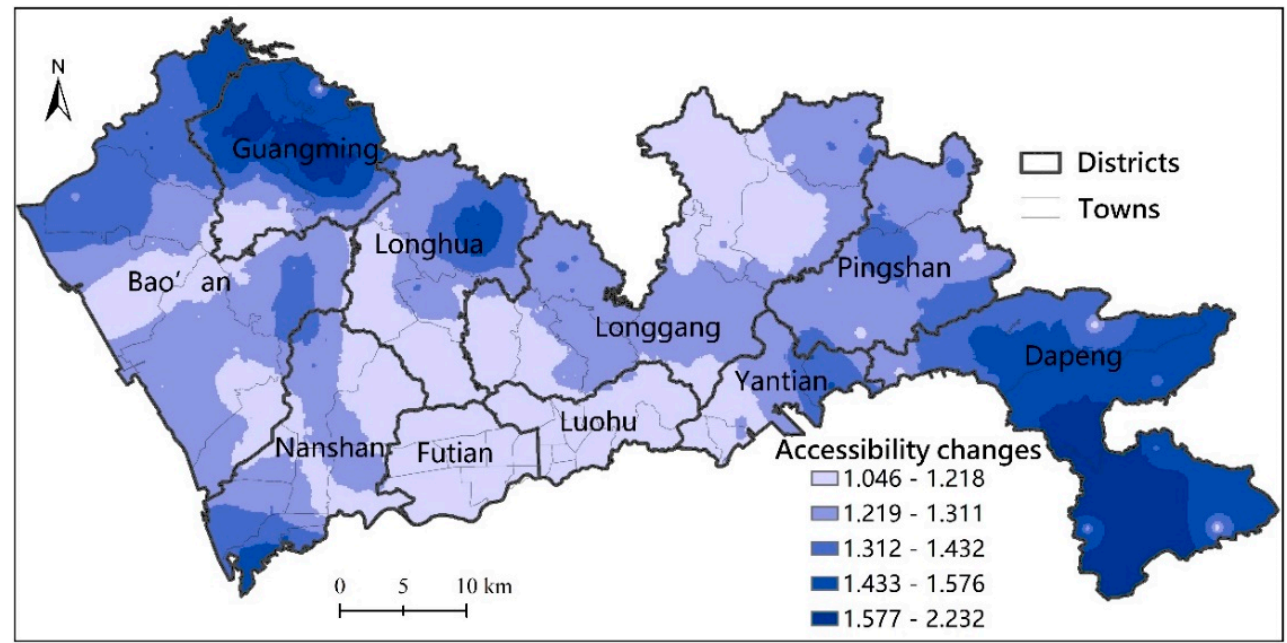

Figure 5. The distribution of improving-efficiency accessibility impacts in Shenzhen.

However, the improvement in equality in healthcare accessibility engendered by improving utilization efficiency is relatively weak. As shown in Figure 6, after considering 
the improving-efficiency accessibility impacts, the distribution of healthcare accessibility is still uneven. The accessibility in northwestern Shenzhen, including Bao'an, Guangming and Longhua, is much poorer than other districts.

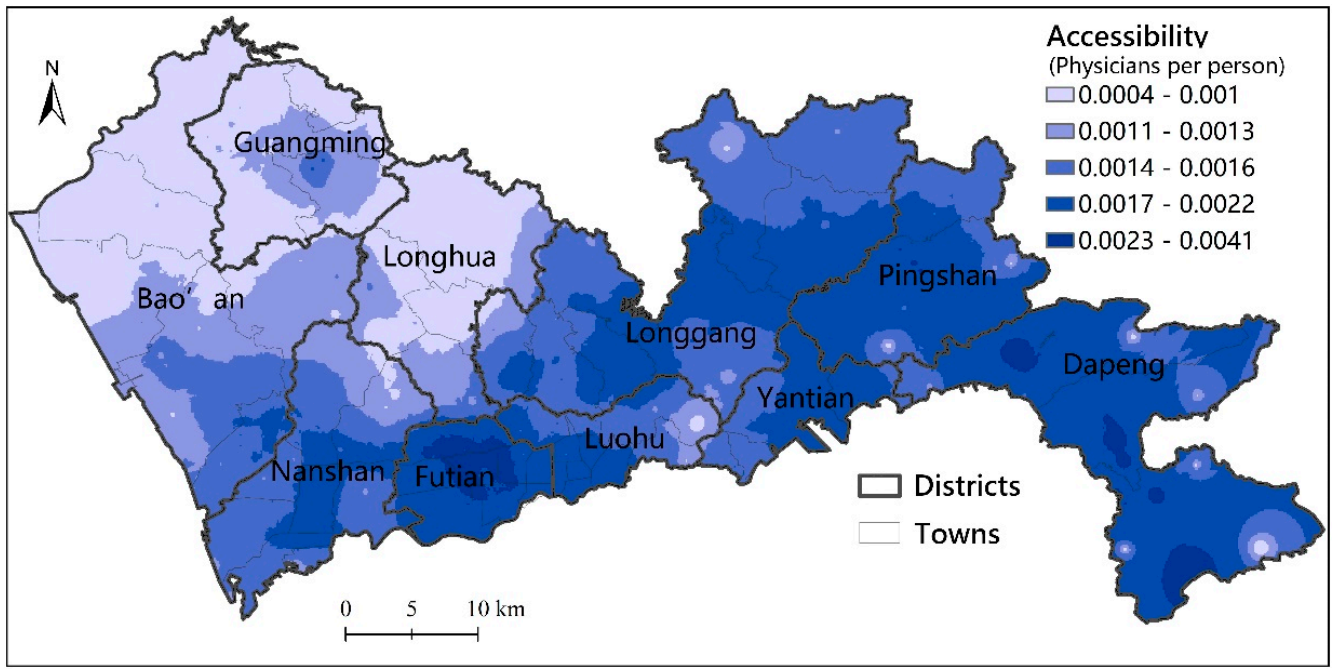

Figure 6. The distribution of healthcare accessibility after improving utilization efficiency in Shenzhen.

\subsection{Adding-Supply Accessibility Impacts of Hierarchical Healthcare System in Shenzhen}

In this part, we further examine the accessibility impacts of adding a certain supply to facilities at different levels. In each scenario, 2000 additional physicians are equally allocated to facilities at each level. Table 3 summarizes relevant statistics that reflect the adding-supply accessibility impacts. With respect to the same amount of supply, when allocated to facilities at a lower level, the increase in average healthcare accessibility would be larger. The accessibility increasing rate by allocating supply at primary facilities is about a half larger than that by allocating supply at tertiary hospitals. This is because lower-level facilities are closer to patients on average. This difference in accessibility impacts cannot be captured by traditional accessibility measures.

Table 3. Adding-supply accessibility impacts of hierarchical healthcare system in Shenzhen.

\begin{tabular}{|c|c|c|c|c|c|c|c|c|c|}
\hline \multirow{2}{*}{$\begin{array}{l}\text { Levels for } \\
\text { Adding } \\
\text { Supply }\end{array}$} & \multicolumn{3}{|c|}{$\begin{array}{c}\text { Weighted Average Overall } \\
\text { Accessibility }\end{array}$} & \multicolumn{3}{|c|}{$\begin{array}{l}\text { Overall Accessibility Inequality } \\
\text { (CV) }\end{array}$} & \multicolumn{3}{|c|}{$\begin{array}{l}\text { Overall Accessibility Inequality } \\
\text { (Gini) }\end{array}$} \\
\hline & I.E. * & $\begin{array}{l}\text { Adding- } \\
\text { Supply }\end{array}$ & Changes & I.E. & $\begin{array}{l}\text { Adding- } \\
\text { Supply }\end{array}$ & Changes & I.E. & $\begin{array}{l}\text { Adding- } \\
\text { Supply }\end{array}$ & Changes \\
\hline Prim & 0.00140 & 0.00159 & $13.6 \%$ & 0.320 & 0.316 & $-1.3 \%$ & 0.178 & 0.175 & $-1.7 \%$ \\
\hline Secondary & 0.00140 & 0.00156 & $11.4 \%$ & 0.320 & 0.320 & 0 & 0.178 & 0.178 & 0 \\
\hline Tertiary & 0.00140 & 0.00153 & $9.3 \%$ & 0.320 & 0.325 & $1.6 \%$ & 0.178 & 0.181 & $1.7 \%$ \\
\hline
\end{tabular}

* Note: I.E. indicates the improving-efficiency scenario. The changes in weighted average accessibility and inequality are calculated in comparison with the improving-efficiency scenario.

As shown in Figure 7, the distributions of accessibility gains by adding supply to facilities at different levels are different. When allocated to primary and secondary facilities, the newly added supply would generate larger accessibility gains in peripheral areas in Shenzhen. This may be good for the promotion of equality in healthcare accessibility. By contrast, when the supply is allocated to tertiary facilities, the accessibility gains are larger in central areas. This would possibly aggravate the inequality in healthcare accessibility. According to changes in the CV and Gini coefficient of accessibility (Table 3), allocating new supply to primary facilities would mitigate the inequality in healthcare accessibility, while allocating supply to tertiary facilities would aggravate inequality. Allocating new supply to secondary facilities has no significant impact on accessibility equality. 


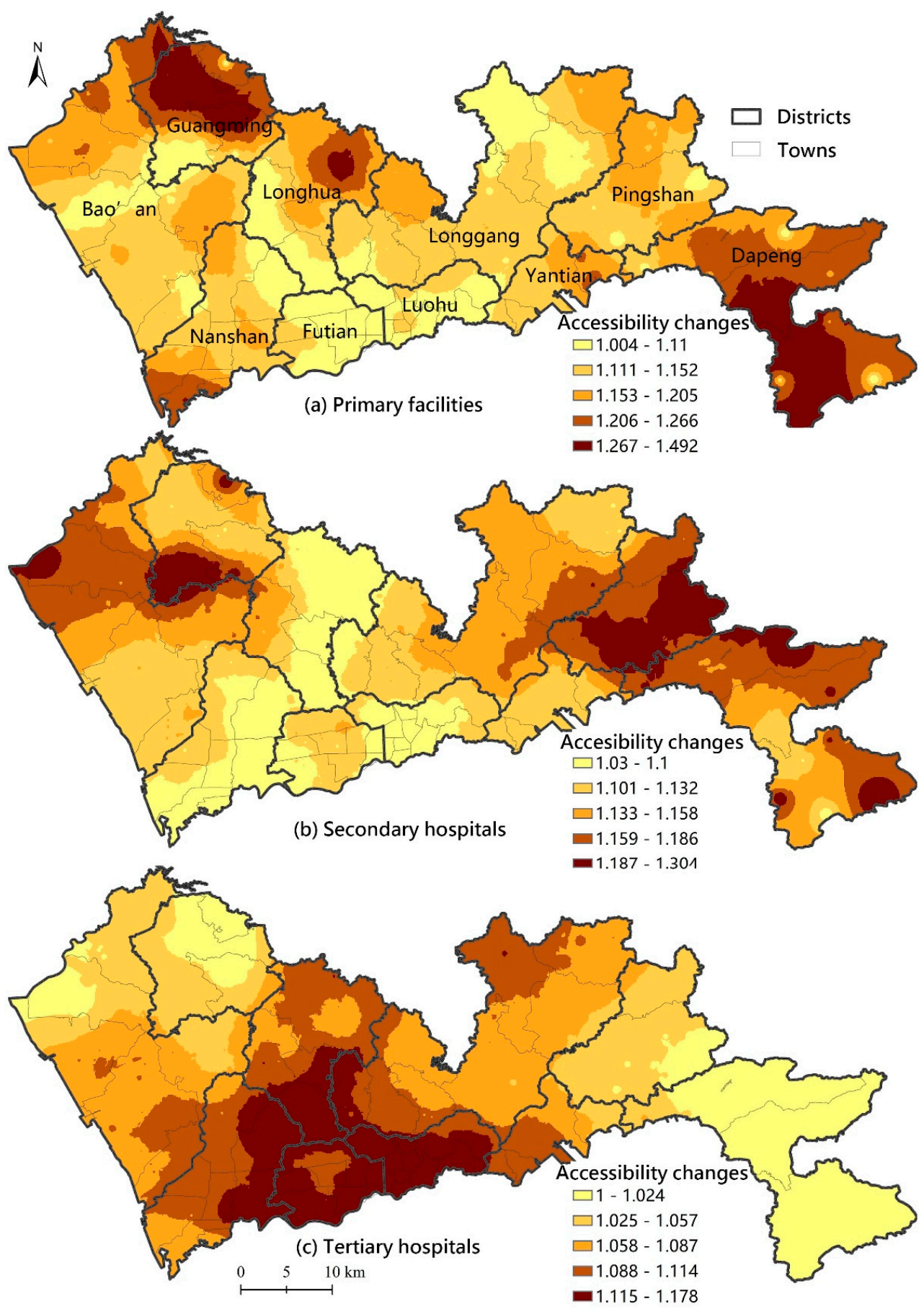

Figure 7. The distribution of accessibility impacts by adding supply at different levels in Shenzhen.

\section{Discussion and Conclusions}

The establishment of a hierarchical healthcare system is highlighted in the "Healthy China 2030" strategy, which is expected to be helpful in promoting equality in healthcare accessibility. However, it still remains unknown how the establishment of a hierarchical healthcare system will impact healthcare accessibility and its equality. Furthermore, traditional spatial accessibility measures are incapable of capturing the differences in accessibility impacts between different facility levels in a hierarchical system.

This study first developed an improved Hierarchical 2SFCA method, which is competent for measuring spatial accessibility to hierarchical healthcare facilities. The method is developed based on the Gaussian 2SFCA, and further incorporates variable catchment 
area sizes, distance friction effects and transport modes with respect to different facility levels. Moreover, a utilization efficiency variable is incorporated into the model to reflect the impacts of a hierarchical healthcare system on accessibility through promoting efficient exploitation of the resources of existing facilities. Taking Shenzhen as a case study, based on the comparison between different scenarios of healthcare accessibility measured by the proposed Hierarchical 2SFCA method, the impacts of the hierarchical healthcare system on healthcare accessibility and its equality were quantitively assessed.

The results reveal that there is significant disparity and inequality in the overall healthcare accessibility in Shenzhen. Furthermore, the magnitude and distribution accessibility are quite different among various facility levels. Lower-level healthcare accessibility is more equally distributed, but is insufficient in magnitude because less resources are provided at lower-level facilities. By contrast, higher-level healthcare facilities provide higher but much more unequal accessibility. To promote the establishment of the hierarchical healthcare system and improve healthcare equality, two complementary countermeasures should be combined. One is to allocate more healthcare resources to primary facilities and improve their medical levels, and the other one is to optimize the locations of higher-level facilities towards more equal distribution.

Focusing on the insufficient utilization of resources at lower-level facilities, this study further examines the impacts of the hierarchical healthcare system on healthcare accessibility and its equality through promoting full utilization of existing facilities. It is estimated that the utilization efficiencies of primary and secondary facilities are only $40 \%$ and $90 \%$ of that of tertiary facilities. The overall healthcare accessibility can be improved by $21.6 \%$ by improving the utilization efficiency of existing facilities. More precisely, the average accessibility to primary and secondary facilities can be improved by $142.9 \%$ and $10.4 \%$, respectively. It is also demonstrated that the improvement in utilization efficiency of existing facilities can improve equality in healthcare accessibility.

Leveraging the improved H2SFCA method, this study also investigates the accessibility impacts of adding a certain new supply of healthcare resources, and compares the differences in such impacts by allocating the new supply to different facility levels. Allocating the same amount of supply to facilities at a lower level can generate larger healthcare accessibility gains. The reason is that lower-level facilities are closer to patients on average and thus can provide better accessibility, which cannot be captured by traditional accessibility measures and proves the effectiveness and superiority of the proposed H2SFCA method. Furthermore, allocating new supply to primary facilities would mitigate the inequality in healthcare accessibility, whereas inequality tends to be aggravated with new supply allocated to tertiary facilities.

This study provides quantitative evidence that the establishment of a hierarchical healthcare system can improve healthcare accessibility and its equality. It also emphasizes the pivotal role of primary facilities in the hierarchical healthcare system. Primary facilities have advantages in their larger amounts and dispersed distribution, and therefore can provide services to the scattered population at a shorter distance. However, the service capacity and medical level of primary facilities are insufficient in the current healthcare system in China, which forces patients to seek healthcare more frequently at higher-level facilities. These findings support the strategies to strengthen primary facilities in the hierarchical healthcare system that have been recommended in the "Guiding opinions on propelling the building of a hierarchical diagnosis and treatment system" and the "Healthy China 2030" planning issued by Chinese governments.

Existing studies have made efforts to decipher the inter-level differences in accessibility to hierarchical healthcare facilities [30,31]. Some studies have also examined the impacts of referrals between facilities at different levels on healthcare accessibility $[13,32,33]$. However, little attention has been paid to the impacts of the insufficient utilization of lower-level facilities on healthcare accessibility. In this regard, this study contributes to the literature by providing transferable methods and procedures for measuring hierarchical healthcare accessibility and assessing the accessibility impacts of the hierarchical healthcare system. 
Notably, the characteristics of the healthcare system investigated in this study may also exist in other developing countries where the hierarchical healthcare system is underdeveloped. Therefore, the findings and proposed methods in this study have possibilities to be applied and further developed in international contexts.

There are some limitations to this study. First, this study mainly focuses on the spatial factors in determining healthcare accessibility. With regard to the differences between accessibility to facilities at different levels, this study mainly considers the advantages of lower-level facilities at closer distances to patients. Future studies can further account for other factors, e.g., the affordability of healthcare services and the economies of scale of higher-level and larger-size facilities. Second, though the improvement in utilization efficiency and the expansion of existing facilities were examined in this study, little has been done to optimize the distribution of hierarchical healthcare facilities. Third, simplifications have been made in this study, i.e., the setting of equal utilization efficiency across facilities at the same level, or the consideration of 2000 additional physicians and equal assignment of this supply in the adding-supply scenarios. Future studies should make further efforts to improve the methodology regarding these simplifications. Fourth, public transport modes (e.g., buses and subway) were not considered in this study due to the difficulty in estimating travel times via multiple modes at the community level.

Author Contributions: Conceptualization, Zhuolin Tao and Wenchao Han; methodology, Zhuolin Tao; software, Zhuolin Tao and Wenchao Han; validation, Zhuolin Tao and Wenchao Han; formal analysis, Zhuolin Tao; investigation, Zhuolin Tao; resources, Zhuolin Tao; data curation, Zhuolin Tao; writing-original draft preparation, Zhuolin Tao; writing — review and editing, Zhuolin Tao and Wenchao Han; visualization, Zhuolin Tao; project administration, Zhuolin Tao; funding acquisition, Zhuolin Tao. All authors have read and agreed to the published version of the manuscript.

Funding: This research was funded by the Key Laboratory of Regional Sustainable Development Modelling, Institute of Geographic Sciences and Natural Resources Research, Chinese Academy of Sciences, grant number KF2020-05.

Institutional Review Board Statement: Not applicable.

Informed Consent Statement: Not applicable.

Data Availability Statement: The datasets used and/or analyzed during the current study are available from the corresponding author on reasonable request.

Acknowledgments: Not applicable.

Conflicts of Interest: The authors declare no conflict of interest.

\section{References}

1. UN. Sustainable Development Goals. 2020. Available online: https://www.un.org/sustainabledevelopment/ (accessed on 14 September 2021).

2. The State Council of the People's Republic of China. “Healthy China 2030" Planning Outline. Beijing, 2016. Available online: http:/ / www.gov.cn/zhengce/2016-10/25/content_5124174.htm (accessed on 3 June 2021). (In Chinese)

3. Wang, F. Measurement, optimization and impact of healthcare accessibility: A methodological review. Ann. Assoc. Am. Geogr. 2012, 102, 1104-1112. [CrossRef]

4. Weiss, D.J.; Nelson, A.; Vargas-Ruiz, C.A.; Gligorić, K.; Bavadekar, S.; Gabrilovich, E.; Bertozzi-Villa, A.; Rozier, J.; Gibson, H.S.; Shekel, T.; et al. Global maps of travel time to healthcare facilities. Nat. Med. 2020, 26, 1835-1838. [CrossRef]

5. Tao, Z.; Cheng, Y. Modelling the spatial accessibility of the elderly to healthcare services in Beijing, China. Environ. Plan. B Urban Anal. City Sci. 2018, 46, 1132-1147. [CrossRef]

6. Zhang, X.; Phillips, R.L.; Bazemore, A.W.; Dodoo, M.S.; Petterson, S.M.; Xierali, I.; Green, L.A. Physician distribution and access: Workforce priorities. Am. Fam. Physician 2008, 77, 1378. [PubMed]

7. Falchetta, G.; Hammad, A.T.; Shayegh, S. Planning universal accessibility to public health care in sub-Saharan Africa. Proc. Natl. Acad. Sci. USA 2020, 117, 31760-31769. [CrossRef] [PubMed]

8. Kim, C.; Tappis, H.; McDaniel, P.; Soroush, M.S.; Fried, B.; Weinberger, M.; Trogdon, J.G.; Lich, K.H.; Delamater, P.L. National and subnational estimates of coverage and travel time to emergency obstetric care in Afghanistan: Modeling of spatial accessibility. Health Place 2020, 66, 102452. [CrossRef]

9. Liu, H.; Fang, C.; Fan, Y. Mapping the inequalities of medical resource provision in China. Reg. Stud. Reg. Sci. 2020, 7, 568-570. 
10. Yu, M.; He, S.; Wu, D.; Zhu, H.; Webster, C. Examining the Multi-Scalar Unevenness of High-Quality Healthcare Resources Distribution in China. Int. J. Environ. Res. Public Health 2019, 16, 2813. [CrossRef] [PubMed]

11. Fang, P.; Zou, X.; Sun, Y. Key points of the hierarchical medical system research in China. Chin. Hosp. Manag. 2014, 34, 1-3. (In Chinese)

12. Jia, P.; Wang, F.; Xierali, I. Delineating Hierarchical Hospital Service Areas in Florida. Geogr. Rev. 2017, 107, 608-623. [CrossRef]

13. Lu, C.; Zhang, Z.; Lan, X. Impact of China's referral reform on the equity and spatial accessibility of healthcare resources: A case study of Beijing. Soc. Sci. Med. 2019, 235, 112386. [CrossRef]

14. Rahman, S.U.; Smith, D.K. Use of Location-Allocation Models in Health Service Development Planning in Developing Nations. Eur. J. Oper. Res. 2000, 123, 437-452. [CrossRef]

15. Tao, Z.; Cheng, Y.; Liu, J. Hierarchical two-step floating catchment area (2SFCA) method: Measuring the spatial accessibility to hierarchical healthcare facilities in Shenzhen, China. Int. J. Equity Health 2020, 19, 164. [CrossRef]

16. Zhou, Z.; Zhao, Y.; Shen, C.; Lai, S.; Nawaz, R.; Gao, J. Evaluating the effect of hierarchical medical system on health seeking behavior: A difference-in-differences analysis in China. Soc. Sci. Med. 2020, 268, 113372. [CrossRef]

17. $\mathrm{Xu}, \mathrm{L} . ; \mathrm{Zhu}, \mathrm{J}$.; Liu, D. Research on modes and strategies for the supply of medical resources in China based on major public health emergencies. City Plan. Rev. 2020, 44, 15-22.

18. Guagliardo, M.F. Spatial accessibility of primary care: Concepts, methods and challenges. Int. J. Health Geogr. 2004, 3, 3. [CrossRef] [PubMed]

19. Levinson, D.M.; Wu, H. Towards a general theory of access. J. Transp. Land Use 2020, 13, 129-158. [CrossRef]

20. Yang, D.; Goerge, R.; Mullner, R. Comparing GIS-based methods of measuring spatial accessibility to health services. J. Med Syst. 2006, 30, 23-32. [CrossRef] [PubMed]

21. Yang, L.; Zhou, J.; Shyr, O.F.; Huo, D.D. Does bus accessibility affect property prices? Cities 2019, 84, 56-65. [CrossRef]

22. Bauer, J.; Groneberg, D.A. Measuring Spatial Accessibility of Health Care Providers-Introduction of a Variable Distance Decay Function within the Floating Catchment Area (FCA) Method. PLoS ONE 2016, 11, e0159148.

23. Chen, X.; Jia, P. A Comparative Analysis of Accessibility Measures by the Two-Step Floating Catchment Area. Int. J. Geogr. Inf. Sci. 2019, 33, 1739-1758. [CrossRef]

24. Luo, W.; Wang, F. Measures of spatial accessibility to health care in a GIS environment: Synthesis and a case study in the Chicago region. Environ. Plan. B Plan. Des. 2003, 30, 865-884. [CrossRef] [PubMed]

25. Luo, W.; Qi, Y. An enhanced two-step floating catchment area (E2SFCA) method for measuring spatial accessibility to primary care physicians. Health Place 2009, 15, 1100-1107. [CrossRef]

26. Dai, D. Black residential segregation, disparities in spatial access to health care facilities, and late-stage breast cancer diagnosis in metropolitan Detroit. Health Place 2010, 16, 1038-1052. [CrossRef] [PubMed]

27. Jamtsho, S.; Corner, R.; Dewan, A. Spatio-Temporal Analysis of Spatial Accessibility to Primary Health Care in Bhutan. ISPRS Int. J. Geo-Inf. 2015, 4, 1584-1604. [CrossRef]

28. Delamater, P.L. Spatial accessibility in suboptimally configured health care systems: A modified two-step floating catchment area (M2SFCA) metric. Health Place 2013, 24, 30-43. [CrossRef]

29. Ni, J.; Liang, M.; Lin, Y.; Wu, Y.; Wang, C. Multi-Mode Two-Step Floating Catchment Area (2SFCA) Method to Measure the Potential Spatial Accessibility of Healthcare Services. ISPRS Int. J. Geo-Inf. 2019, 8, 236. [CrossRef]

30. Jin, M.; Liu, L.; Tong, D.; Gong, Y.; Liu, Y. Evaluating the Spatial Accessibility and Distribution Balance of Multi-Level Medical Service Facilities. Int. J. Environ. Res. Public Health 2019, 16, 1150. [CrossRef]

31. Zhang, S.; Song, X.; Wei, Y.; Deng, W. Spatial Equity of Multilevel Healthcare in the Metropolis of Chengdu, China: A New Assessment Approach. Int. J. Environ. Res. Public Health 2019, 16, 493. [CrossRef] [PubMed]

32. Yang, N.; Shen, L.; Shu, T.; Liao, S.; Peng, Y.; Wang, J. An integrative method for analyzing spatial accessibility in the hierarchical diagnosis and treatment system in China. Soc. Sci. Med. 2020, 270, 113656. [CrossRef]

33. Xiao, Y.; Chen, X.; Li, Q.; Jia, P.; Li, L.; Chen, Z. Towards healthy China 2030: Modeling health care accessibility with patient referral. Soc. Sci. Med. 2021, 276, 113834. [CrossRef] [PubMed]

34. The State Council of the People's Republic of China. Guiding Opinions on Propelling the Building of a Hierarchical Diagnosis and Treatment System. Beijing, 2015. Available online: http://www.gov.cn/zhengce/content/2015-09/11/content_10158.htm (accessed on 11 June 2021). (In Chinese)

35. Farahani, R.Z.; Hekmatfar, M.; Fahimnia, B.; Kazemzadeh, N. Hierarchical facility location problem: Models, classifications, techniques, and applications. Comput. Ind. Eng. 2014, 68, 104-117. [CrossRef]

36. Sahin, G.; Süral, H. A review of hierarchical facility location models. Comput. Oper. Res. 2007, 34, 2310-2331. [CrossRef]

37. Shenzhen Municipal Health Commission. List of Healthcare Facilities in Shenzhen. 2021. Available online: http:/ /wjw.sz.gov.cn/ bmfw/wycx/fwyl/yycx/index.html (accessed on 16 December 2020). (In Chinese)

38. Shenzhen Municipal Health Commission. The Summary of Health Statistics 2015 of Shenzhen. 2020. Available online: http: //wjw.sz.gov.cn/xxgk/tjsj/zxtjxx/content/post_7786068.html (accessed on 14 September 2021). (In Chinese)

39. Shenzhen Municipal Health Commission. The 2013 Yearbook of Health Statistics of Shenzhen. 2014. Available online: http: //wjw.sz.gov.cn/xxgk/tjsj/zxtjxx/content/post_3167134.html (accessed on 14 September 2021). (In Chinese)

40. Shen, Q. Location characteristics of inner-city neighborhoods and employment accessibility of low-wage workers. Environ. Plan. B Plan. Des. 1998, 25, 345-365. [CrossRef] 
41. Cheng, L.; Yang, M.; De Vos, J.; Witlox, F. Examining geographical accessibility to multi-tier hospital care services for the elderly: A focus on spatial equity. J. Transp. Health 2020, 19, 100926. [CrossRef]

42. Tao, Z.; Yao, Z.; Kong, H.; Duan, F.; Li, G. Spatial accessibility to healthcare services in Shenzhen, China: Improving the multi-modal two-step floating catchment area method by estimating travel time via online map APIs. BMC Health Serv. Res. 2018, 18, 345. [CrossRef] [PubMed]

43. Wang, F.; Xu, Y. Estimating O-D travel time matrix by Google Maps API: Implementation, advantages, and implications. Ann. GIS 2011, 17, 199-209. [CrossRef]

44. TsingHua Urban Planning and Design Institute (THUPDI); Mobike Technology Co. Ltd. (Mobike). The 2017 White Paper on Shared Bikes and Urban Development. 2017. Available online: https://www.sohu.com/a/133766880_585110 (accessed on 8 June 2020). (In Chinese).

45. Cheng, G.; Zeng, X.; Duan, L.; Lu, X.; Sun, H.; Jiang, T.; Li, Y. Spatial difference analysis for accessibility to high level hospitals based on travel time in Shenzhen, China. Habitat Int. 2016, 53, 485-494. [CrossRef] 